\title{
La huerta escolar: estrategia pedagógica apoyada en las tecnologías de la información y la comunicación para el desarrollo de competencias investigativas ${ }^{1}$
}

\section{The school garden: pedagogical strategy supported by information and communication technologies for the development of research skills}

DOI: http://dx.doi.org/10.17981/cultedusoc.9.3.2018.59

\author{
Artículo de investigación. Fecha de recepción: 15/06/2018. Fecha de aceptación: 27/11/2018 \\ Vilma Jiménez² \\ Javier Sierra Martinez; Ketty Padilla Hormechea; Yadira Sierra Soraca; \\ Marlene Narvaez; Myriam Caro Torres y Vilma Jimenez ${ }^{3}$ \\ IED Celinda Mejía López del corregimiento de Barroblanco-Magdalena (Colombia) \\ vilmaj1@hotmail.com
}

Para citar este artículo:

Jiménez, V., Sierra, J., Padilla, K., Sierra. Y., Narvaez, M., Caro, M. y Jimenez, V. (2018). La huerta escolar: estrategia pedagógica apoyada en las tecnologías de la información y la comunicación para el desarrollo de competencias investigativas. Cultura. Educación y Sociedad 9(3), 499-504. DOI: http://dx.doi.org/10.17981/cultedusoc.9.3.2018.59

\section{Resumen}

El presente artículo presenta los resultados derivados de la huerta escolar como estrategia pedagógica para mejorar el proceso investigativo en los estudiantes, surge ante la necesidad presente en la Institución Educativa Departamental (IED) Celinda Mejía López del corregimiento de Barroblanco Magdalena, debido a la dificultad con relación a su rendimiento académico. También la investigación busca utilizar la huerta escolar como estrategia para fomentar interés investigativo en los estudiantes y con el ánimo de transformar su realidad educativa y social, se traza como objetivo el fomentar las competencias investigativas de los estudiantes por medio de la estrategia de la huerta escolar con apoyo en las tecnologías de la información y comunicación. Con base a las encuestas aplicadas se logró evidenciar que un porcentaje significativo de la población estudiantil desconoce elementos básicos de la huerta escolar, así como también desconocen aspectos relacionados con la investigación.

Palabras clave: Tecnologías de la Información y la Comunicación (TIC), huerta escolar, competencias investigativas.

\section{Abstract}

This article presents the results derived from the school garden as a pedagogical strategy to improve the investigative process in the students, arises from the present need in the Departmental Educational Institution (IED) Celinda Mejía López from the district of Barroblanco - Magdalena, due to the difficulty in relation to his academic performance. The research also seeks to use the school garden as a strategy to promote research interest in students and with the aim of transforming their educational and social reality, the objective is to promote the research skills of students through the strategy of the school garden with support in information and communication technologies. Based on the surveys applied, it was possible to demonstrate that a significant percentage of the student population does not know basic elements of the school garden, as well as unaware aspects related to the research.

Keywords: Information and Communication Technologies (ICT), school garden, investigative skills.

1 Este artículo ha sido derivado del Proyecto Fortalecimiento de la Cultura Ciudadana y Democrática en CT+I a través de la IEP apoyada en TIC en el Dpto. del Magdalena.

2 Líder del grupo de investigación.

3 Docentes de la institución Educativa Departamental Celinda Mejía López del corregimiento de Barroblanco-Magdalena

- The author; licensee Universidad de la Costa - CUC.

Cultura, Educación y Sociedad vol. 9 no. 3, pp. 499-504. Diciembre, 2018

Barranquilla. ISSN 2389-7724 Online 


\section{Introducción}

La Institución Educativa Celinda Mejía López del corregimiento de Barroblanco Magdalena, pertenece a una comunidad rural donde la mayoría de sus estudiantes presentan un bajo rendimiento académico y poco interés hacia la investigación. Sus experiencias formativas se ven mayormente afectadas por los escasos recursos académicos que poseen, tanto en la escuela como en sus hogares. Entre estas deficiencias se encuentran:

- No cuentan con libros de consulta en sus hogares,

- No tienen el hábito para investigar lo desconocido,

- En la institución educativa, la conectividad a internet es irregular,

- No hay servicio de biblioteca en las horas de la tarde (no se cuenta con bibliotecaria, ni secretaria),

- Por parte de los docentes no se ejecutan proyectos donde los estudiantes participen en la investigación. Solo se hacen consultas de determinados temas.

- A nivel nacional y departamental, se han realizado proyectos de capacitación a los docentes en cuanto a la investigación.

Teniendo en cuenta lo anterior, surge en el grupo de investigación la inquietud sobre cuáles serán las implicaciones que podría tener la vinculación de la estrategia de la huerta escolar en los estudiantes, tanto en el desarrollo de sus procesos de aprendizajes, como en la visión que manejan de sí mismos y de su entorno, propiciando así el interés investigativo en los mismos. Entendiendo estrategia como un dinamismo que potencializa el crecimiento socioeconómico, que genera desarrollo e inclusión (Pacheco, 2013)

Así entonces, las organizaciones, señalan Mendoza, López y Salas (2016), "son entes que generan sus propios objetivos y diseñan la forma como alcanzarlos teniendo en cuenta, necesariamente, las influencias del entorno social" (p. 62). Las escuelas no escapan de ello y es de allí que, en las organizaciones cualquiera que sea su ámbito, se hace preciso introducir una gestión estratégica como una preocupación por posicionarse (Mendoza, López y Salas, 2016)

El presente proyecto de investigación tiene como objetivo fomentar en los estudiantes experiencias significativas (Ausubel, 2001), utilizando la Investigación como Estrategia Pedagógica (IEP), por ser una herramienta útil y valiosa en el trabajo con estudiantes, para hallar resultados satisfactorios en los distintos niveles de desempeño de los mismos (Restrepo, 2009; Villalba, 2012; Mejía y Manjarréz, 2010, 2011; Orozco, 2015); teniendo en cuenta las capacitaciones direccionadas por el Ministerio de Educación Nacional y la Secretaría de Educación del departamento del Magdalena, a través del programa ciclón y la Universidad de la Costa CUC, dirigido a los docentes de las instituciones educativas oficiales, en aras de mejorar la labor docente y su quehacer pedagógico.

La investigación como estrategia debe ser concebida similar al pensamiento estratégico que según Arellano (2004) citado por Mendoza y López (2015), "hace referencia a la forma del pensamiento que se dirige hacia la creatividad, imaginación, y búsqueda de opciones innovadoras y realistas, como potenciación de lo posible enmarcado dentro de un proyecto de construcción intencional de la realidad". (p. 154)

El grupo de investigación conformado por los docentes de primaria, dirige el proyecto aprovechando los recursos disponibles en la institución, se desarrolló la huerta escolar en el terreno de la escuela, teniendo en cuenta la articulación con las herramientas que ofrecen las Tecnologías de la Información y la Comunicación (TIC). 
Con lo cual "se podrá avanzar en el conocimiento sobre las condiciones de creación y de permanencia, y de manera más amplia, del dinamismo del sistema social". (Maculan, Jiménez, \& Castellanos, 2015, p. 16)

\section{El huerto}

También conocido como "huerta", es un espacio de terreno donde se pueden sembrar algunos tipos de plantas útiles para el consumo humano, sean estas medicinales, alimenticias, como verduras, frutas, vegetales, y en muchas ocasiones árboles frutales (Olguin, 2011; Escrivá, 2007; Stojanov, 2003;). Cuando este espacio se encuentra en la institución educativa, se le llama huerto escolar.

\section{La huerta escolar}

Son pequeños espacios de las instituciones educativas cuyo objetivo primordial es que el niño o niña o los jóvenes lleguen a experimentar el mundo de relaciones interdependientes que hay entre las plantas y su medio, observan los cambios que sufren por influencia de la luz, el agua, el suelo, la temperatura que son los factores físicos que intervienen en su crecimiento y su desarrollo (Organización de las naciones unidas para la Agricultura y la alimentación-FAO, 2006).

La huerta escolar es una estrategia que ha sido utilizada en las instituciones educativas, con el fin de trabajar diversos aspectos en los estudiantes relacionados con la motivación, relaciones sociales, competencias investigativas; entre otras, teniendo como resultado receptividad por parte de los mismos. Entre estos resultados se encuentra que los estudiantes que trabajaron la huerta escolar, presentaron un desarrollo positivo en sus áreas cognoscitivas, se fomentan valores como el respeto hacia la naturaleza y presentan un mejor desempeño en el aspecto social (Polanco y Moreno, 2013; Moreno y Nieves, 2014; Botella,
Hurtadoy Cantó, 2014) (Saavedra Sueldo, Urrutia, Paravié, Rohvein, \& Corres, 2014). Se evidencia, con base en lo anterior, que la huerta escolar es una herramienta didáctica con gran potencial emotivo y motivacional que apoya el fomento del interés hacia lo académico, personal e investigativo en la escuela.

Así las cosas, es importante destacar la articulación entre la estrategia del desarrollo de la huerta escolar y el uso de las TIC para el éxito de este proyecto; ya que son una herramienta que facilita la práctica pedagógica y promueve la interacción y la enseñanza- aprendizaje en estudiantes y docentes, directivos, padres de familia y la comunidad (Herrera, 2016; Cabero, 2012).

\section{Metodología}

El artículo que se presenta es un producto derivado del estudio de la huerta escolar como estrategia pedagógica apoyada en las tecnologías de la información y la comunicación para el desarrollo de competencias investigativas. Desarrollado en la Institución Educativa Departamental (IED) Celinda Mejía López del corregimiento de Barroblanco - Magdalena; su objetivo principal ha sido utilizar la huerta escolar como estrategia para fomentar interés investigativo en los estudiantes.

El estudio contempla el siguiente proceso: El diseño se realizó bajo el paradigma de la investigación cualitativa, la cual estudia la realidad tal como sucede, tal y como sucede, para extraer el sentido, comprender e interpretar los fenómenos y las percepciones de las personas implicadas (Ruiz, 2012; Pérez, 2002; Hernández, Fernández y Baptista, 2014). Se utilizó el modelo de investigación acción participativa, por ser una propuesta metodológica que involucra a los actores en el conocimiento y la solución de sus problemas, generando conjuntamente los conocimientos para definir las acciones adecuadas y lograr cambios (Martínez, 2004). 
La unidad de análisis fue conformada por estudiantes de tercero de primaria de la Institución Educativa Departamental (IED), Celinda Mejía López del corregimiento de Barroblanco - Magdalena. Para recoger los datos se utilizaron, encuestas y observación directa.

Con base a las encuestas aplicadas se logró evidenciar que un porcentaje significativo de la población estudiantil desconoce elementos básicos de la huerta escolar, así como también desconocen aspectos relacionados con la investigación.

\section{Resultados}

Con base a lo desarrollado en el proyecto investigativo, se obtuvo los siguientes resultados:

El $60 \%$ de los estudiantes saben lo que es una huerta escolar, mientras que el $40 \%$ no.

El $50 \%$ de los estudiantes sabe cómo cuidar una huerta escolar y el otro $50 \%$ no.

El $80 \%$ de los estudiantes no han tenido una huerta escolar, mientras que el $20 \%$ sí.

EL $60 \%$ de los estudiantes no conoce que plantas se pueden sembrar en la huerta escolar y solamente el $40 \%$ si conoce que plantas se pueden sembrar.

El $57 \%$ de los estudiantes dicen que la huerta escolar no beneficia a la comunidad, mientras que el $43 \%$ dicen que sí.

El $89 \%$ de los estudiantes si conoce la planta de ají y solamente el $11 \%$ no.

El $50 \%$ de los estudiantes si ha observado el proceso de germinación de una planta y el otro $50 \%$ no lo ha observado.

El $77 \%$ de los estudiantes sabe cómo cuidar una planta mientras que el $23 \%$ no.

EL $87 \%$ de los estudiantes no han investigado sobre algún tipo de planta y solamente el $13 \%$ si ha investigado.

\section{Conclusiones}

A partir de la investigación realizada, se concluye que como estrategia de enseñanza aprendizaje, la huerta escolar resulta positiva ya que los estudiantes aprenden desde la experiencia, fortalecen la participación y colaboración y se propicia un diálogo de saberes entre las personas que intervienen, por lo que el proceso de enseñanza-aprendizaje se torna bidireccional y significativo para los participantes del proceso.

Es importante rescatar varios aspectos de la misma investigación, como lo es, la innovación y la implementación de estrategias motivadoras para el aprendizaje ameno del estudiante dentro y fuera del aula.

Desde todo punto de vista, los cambios a estrategias de este tipo, favorecen de manera eficaz el desarrollo del aprendizaje de muchos estudiantes, ya que el comportamiento pro ambiental está encaminado a ser innovador junto a las herramientas tecnológicas, lo que resulta una combinación pertinente en los procesos de enseñanza.

También es pertinente plantear que, ciertos aspectos sobre el uso de la huerta como estrategia pedagógica y su relación con una conducta pro-ambiental, produce en los estudiantes, puede ilustrarse con lo expresado en distintas posturas teóricas sobre el tema:

1. Hernández e Hidalgo (1998), define la conducta pro-ambiental como "conducta protectora del ambiente" o "Conducta pro ecológica". También se le concibe como "conducta ambiental responsable", "conducta ecológica responsable" y "conducta ambiental amigable" (Bustos, 2004). Sin embargo, cabe destacar que la variedad de términos empleados se debe a una variedad de enfoques; por ejemplo para algunos autores, la Conducta Proambiental (CPA), es un hábito; para otros es una conducta intencional y dirigida; y algunos más indican que solamente puede surgir de manera forzada. 
2. Es fundamental precisar el término de CPA dentro de la investigación, al ser una definición que debe precisarse, de acuerdo con las necesidades del contexto donde se esté interactuando. Las propuestas de intervención ambiental inadecuadas pueden ocurrir porque no se ha definido claramente la conducta - meta en estudiantes, conducta que con frecuencia en educación tiende a tener una definición genérica, razón por la cual se presentan lagunas en la claridad de sus objetivos.

3. Para Corral-Verdugo, Frías, Pérez, Orduña y Espinoza (2002), la conducta proambiental es el resultado de la preservación de los recursos naturales y/o de la reducción del deterioro, debe ser intencional y desplegar habilidades concretas como la anticipación del resultado de la acción, deliberación para actuar y direccionar las conductas hacia metas concretas, relacionadas en un contexto con requerimientos específicos y en relación a la conciencia ecológica.

Conociendo los conceptos anteriores, lo importante es la conducta-meta que se establezca en la implementación de estrategias, en toda estrategia investigativa que se trabaje con los estudiantes en el aula se debe tener claro o saber de dónde se parte y hasta donde se pretende llegar; por ejemplo, la viabilidad de la huerta como estrategia pedagógica, por ende el innovar procesos o estrategias encaminadas al comportamiento ambiental, facilitar y ensayar los aspectos pedagógicos que poco se desarrollan dentro del aula, ya que no son tan recurrentes en algunas instituciones.

Una de las expresiones comunes que debemos evitar en nuestros proyectos de mejoramiento ambiental es la utilización de discursos generalizados sobre la crisis ecológica porque se refieren a problemas de la humanidad y, por ende, del comportamiento humano. Conviene entonces precisar cuáles son los comportamientos de las personas los que provocan un incremento de la gravedad de un problema ambiental en particular.
Toda esta intervención creativa que pretendemos poner en práctica como docentes, debe partir de entender de dónde nacen las necesidades de crear estrategias de este tipo y con qué apoyo de herramientas. En la cultura de la organización escolar, en los medios de comunicación, en las redes sociales, en las aulas y fuera de ellas, en la comunidad, debemos asumir el desafío de entender que todo lo que nos da el contexto o la naturaleza puede ser potencializado con el fortalecimiento de los procesos de aprendizaje, el trabajar con calidad y sobre todo seguir desarrollando competencias investigativas para transformar responsablemente la sociedad.

\section{Referencias}

Ausubel, D. (2001). Teoría del aprendizaje significativo. Fascículos de CEIF, 1, 1-10.

Botella, A., Hurtado, A. y Cantó, J. (2014). Las competencias básicas a través del huerto escolar: una propuesta de proyecto de innovación. En J. Maquilón y N. Orcajada (Eds.), Investigación e innovación en formación del profesorado (173-182). Murcia: Editum.

Bustos, A. (2004). Modelo de conducta proambiental para la conservación de agua potable. [Tesis de doctorado]. Universidad Nacional Autónoma, México.

Cabero, J. (2012). El rol del profesor ante las nuevas tecnologías de la información y la comunicación. Agenda académica, 7(1), 41-57.

Corral-Verdugo, V., Frías, A., Pérez, U., Orduña, C. y Espinoza, G. (2002). Consumo doméstico de agua, motivación para ahorrarla, y la continua tragedia de los comunes. En, V. Corral-Verdugo, Conductas protectoras del ambiente. (81-97). México, D.F.: Conacyt.

Escrivá, M. (2007). Huerta orgánica. Buenos Aires: Albatros.

FAO. (2006). Crear y manejar un huerto escolar: Recuperado de www.fao.org/3/aa0218s.pdf 
Herrera, A. y Herrera, A. (2016). Las TIC como herramientas pedagógicas. Recuperado de http://aracelisherrera10.blogspot.com.co/

Maculan, A., Jiménez, C. y Castellanos, O. (2015). Aprendizaje en el proceso de incubación de empresas de base tecnológica. Económicas CUC, 36(1), 15-48.

Martínez, M. (2004). Ciencia y arte en la metodología cualitativa. México, D.F: Trillas

Martínez, J. (2004). Comportamiento proambiental. Una aproximación al estudio del desarrollo sustentable con énfasis en el comportamiento persona-ambiente. Theomai, 99. Recuperado de http://www. redalyc.org/articulo.oa?id=12499303

Mendoza, D. y López, D. 2015). Pensamiento estratégico: centro neurálgico de la planificación estratégica que transforma la visión en acción. Económicas CUC, 36(1), 153-179.

Mendoza, D., López, D. y Salas, E. (2016). Planificación estratégica de recursos humanos: efectiva forma de identificar necesidades de personal. Económicas CUC, 37(1), 61-79.

Mejía, M. y Manjarrés M. (2010). Las pedagogías fundadas en la investigación. Búsquedas en la reconfiguración de la educación. +Revista Internacional Magisterio, 42. 16-26.

Mejía, M. y Manjarrés, M. (2011). La investigación como estrategia pedagógica una apuesta por construir pedagogías críticas en el siglo XXI. Praxis \& Saber, 2(4), 127. http://dx.doi. org/10.19053/22160159.1127

Moreno, T. y Nieves Q. (2014). La huerta escolar como medio de integración curricular de las áreas fundamentales e insumo de orientación a las prácticas pedagógicas. [Tesis]. Universidad del Tolima, Ibagué. Recuperado de http:// repository.ut.edu.co/handle/001/1472
Olguin, S. (2011). Qué es una huerta orgánica. Recuperado de http://www.innatia. com/s/c-huerta-organica/a-que-es-huertaorganica.html

Orozco, M. (2015). Métodos, formas, procedimientos y sistemas de enseñanza en una concepción de la pedagogía como arte según José M. Zamora. Aula, 21(0), 165. h t tp://dx.doi.org/10.14201/ aula201521165176

Pacheco, C. M. (2013). Estrategias empresariales más utilizadas por las pymes en Sincelejo. Económicas CUC, 34(1), 183202.

Pérez, G. (2002). Investigación cualitativa. Retos e interrogantes. II técnicas y análisis de datos. Madrid: La Muralla.

Polanco, L. y Moreno, A. (2013). El huerto educativo como medio de enseñanza de contenidos curriculares para niños/as de 6 años de edad del año lectivo 2012-2013. [Tesis Licenciatura]. Universidad Politécnica Salesiana, Quito.

Restrepo, B. (2009). Investigación de aula: formas y actores. Recuperado de http:// bibliotecadigital.udea.edu.co/bits $\mathrm{t} \mathrm{r}$ e a m / $10495 / 3498 / 1 /$ $\mathrm{R}$ e s t r e p o B e r n a r d o _ 2009 Investigaci\%C3\%B3naulasformas.pdf

Ruiz, J. (2012). Metodología de la investigación cualitativa. Bilbao: Universidad de Deusto.

Saavedra, C., Urrutia, S., Paravié, D., Rohvein, C. y Corres, G. (2014). Una propuesta metodológica paraladeterminación de capacidades estratégicas en pymes industriales. INGE CUC, 10(2), 43 - 50. Recuperado de https://52.0.212.120/ingecuc/article/view/489

Stojanov, E. (2003). Huerta orgánica. Buenos Aires: Reysa Ediciones.

Villalba, A. (2012). Dos paradigmas contrapuestos en la evaluación educativa, dos formas diferentes de concebir al alumno. Aula Universitaria, 1(14). http://dx.doi. org/10.14409/au.v1i14.4123 